\title{
The stress ratio effect on plastic dissipation during fatigue crack growth
}

\author{
H. Quan ${ }^{1, *}$, R.C. Alderliesten ${ }^{1}$, and R. Benedictus ${ }^{1}$. \\ ${ }^{1}$ Structural Integrity \& Composites, Faculty of Aerospace Engineering, TU Delft, Netherlands
}

\begin{abstract}
Plastic energy dissipation is inevitable during fatigue crack growth. There have been previous attempts reported in literature to correlate the plastic dissipated energy $(d W / d N)$ to fatigue crack growth rate $(d a / d N)$. However, at a given $d W / d N$, the $d a / d N$ changes with the ratio of minimum and maximum loads, known as the stress ratio. This paper describes an experimental study carried out on 2024-T3 central crack tension specimens to quantify the relation between $d W / d N$ and $d a / d N$. By selecting different stress ratios in the individual tests, the experiments reveal the influence of the stress ratio on this relationship. It is evident that $d W / d N$ has no unique relationship with $d a / d N$ valid for the tested stress ratios. Instead, the relationship for each stress ratio is different. This is illustrated with the value of plastic dissipation per unit of fatigue crack growth $(d W / d a)$, representing the effective resistance to the crack increment. This value is not a constant, but changes with the stress ratios and $d a / d N$ values. Hence the plastic energy dissipation cannot be used directly for predicting crack growth.
\end{abstract}

\section{Introduction}

Fatigue failure is a major failure type in aerospace engineering, so it is important to have a deep understanding of this failure type, in order to mitigate it. Although Linear Elastic Fracture Mechanics (LEFM) has been applied successfully to practical engineering fatigue problems through the use of the Paris relation, LEFM still fails in explaining the physical nature of fatigue phenomena in metallic materials. This is because metallic materials are ductile, and the plasticity phenomena strongly influences the fatigue crack growth rate $(d a / d N)$. Therefore, if a deeper understanding of the nature of fatigue is needed, it has to be answered how plasticity influences fatigue crack propagation in metallic materials. In this paper, the fatigue crack phenomena will be discussed from a physical perspective. An energy approach is chosen because the energy approach shows the universality among various materials and the plasticity itself represents a typical type of energy dissipation.

There is literature [1-17] on the topic of the plastic energy dissipation during the fatigue crack propagation. Most of the papers aim at finding the relation between the plastic energy dissipation per cycle $(d W / d N)$ and $d a / d N$, and their work could be summarized as:

$$
\frac{d W}{d N}=\frac{d a}{d N} \frac{d W}{d a}
$$

The $d W / d a$ represents the plastic energy dissipated per unit fatigue crack extension. There are some differences among the studies mentioned above, mainly in the definitions and in the method of obtaining those values of $d W / d N$ and $d W / d a$. Klingbeil et al. [1-6] relate the fatigue crack growth to the static crack propagation and claim that $d W / d a$ is a material property and its value should be equal to the static fracture toughness $\mathrm{G}_{\mathrm{c}}$. Then, $d W / d N$ is the total plastic dissipation energy per cycle per unit width. Karlsson et al.[7-11] use the finite element method to predict $d a / d N$. They believe that the fatigue crack propagation is the result of the degradation in a process zone ahead of the crack tip where the plastic deformation occurs. So Karlsson et al.[7-11] link $d a / d N$ with the plastic dissipation in the process zone, which is represented by the reverse cyclic plastic zone ahead of the crack tip. Karlsson et al. [7-11] believe $d W / d a$ should be a constant that is not necessarily equal to the value of $\mathrm{G}_{\mathrm{c}}$. Ranganathan et al. [12-13] perform an experimental investigation on the relation between the total energy dissipated per cycle and $d a / d N$. Their experimental results show that the total energy dissipated per unit fatigue crack extension $(d W / d a)$ is not a constant and could depend on the different mechanism of crack growth. Zheng [14] simulates the fatigue crack propagation based on the plastic dissipation value of critical distances. Smith [15] assumes that the energy dissipation to create a unit of new crack surface could be considered as a material property and applies this assumption to simulate fatigue crack growth with 3D finite element model. Besel et al. [16-17] study AA2024T3 plastic dissipation with digital image correlation (DIC) method and coupled FE simulation. From the work mentioned above, although with some slight differences, there seems to be a contradiction between the work of [1-6,15] and [12-13]: some researchers seem to believe that the $d W / d a$ value is a material property,

Corresponding author: h.quan@tudelft.nl 
but the others disagree with this. Therefore the research questions in this paper are as follows:

1. Is the value $d W / d a$ (it is defined as the total plastic dissipation per unit fatigue crack growth) a material property that is approximately constant?

2 . Does the same value of $d W / d N$ correspond to the same $d a / d N$ among various stress ratios?

\section{EXPERIMENT METHODOLOGY}

\subsection{Fatigue experiments description}

In order to obtain the plastic energy dissipation during fatigue crack growth, Mode I fatigue crack propagation experiments of the 2024-T3 aluminium alloy CCT specimens were carried out. The geometry of the specimen is shown in Fig. 1. The thickness of 2024-T3 specimens was $4 \mathrm{~mm}$. The initial crack was made in 3 steps: first a small hole was drilled at the centre of the plate; then on each side of the hole a small notch was created by fret saw; finally the fatigue cracks were produced after fatigue precracking.

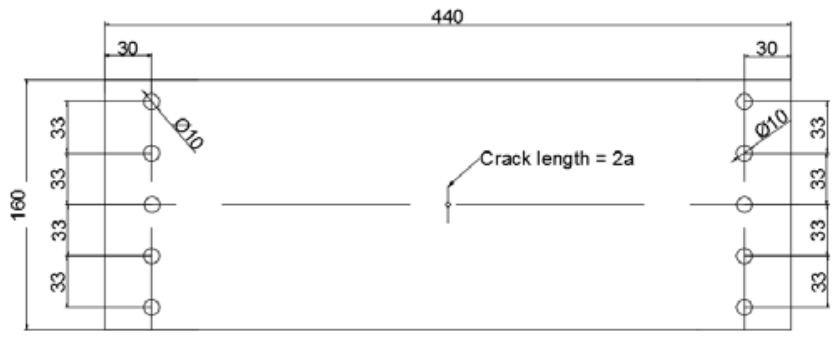

Fig. 1. The CCT specimens(The unit is $\mathrm{mm}$ )

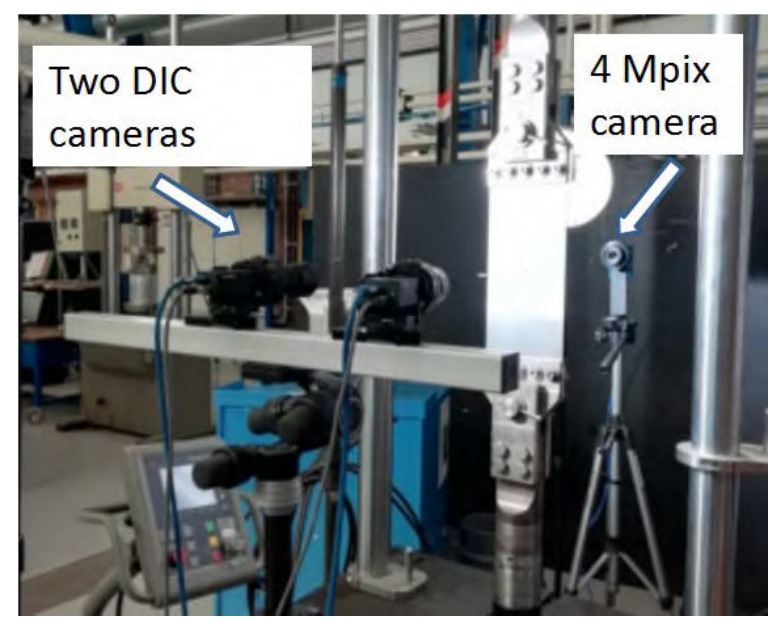

Fig. 2. The set-up of the experiments

During the fatigue experiments, there is one single 4 Mpix camera on one side of the specimens in order to measure the fatigue crack length visually after the experiment. The other side of the specimens were painted with a speckle pattern for the purpose of measuring the strain and displacement field with DIC method. For the DIC measurement, a pair of $80 \mathrm{~mm}$ lenses cameras were used to observe the deformation of the test specimens. The set-up is shown in Fig. 2.

The fatigue tests were carried out under a combination of baseline cycles and slow cycles. The frequency of baseline cycles is $10 \mathrm{~Hz}$. The slow cycles were used to make measurements and take photos, because the camera system needed some time to capture images. In the fatigue experiments, the slow cycles were used after every 2000-3000 baseline cycles. The specimens were tested at a load controlled condition with MTS $250 \mathrm{kN}$ fatigue machine. For the stress ratio of $R=0.05$ the maximum load is $45 \mathrm{kN}$, while for $R=0.5$ the maximum load is $60 \mathrm{kN}$.

\subsection{Data acquisition and post-processing}

The photos taken by 4 Mpix camera during the fatigue experiments were used to measure the crack length at the number of cycles the image was captured, which was evaluated to obtain $d a / d N$. The photos taken for DIC were processed with DIC post-processing software.

The energy dissipation per cycle during the fatigue experiments was determined by the integration of the hysteresis loop of the load-displacement curve measured throughout an entire fatigue load cycle. The load values were obtained by the load cell of the fatigue machine, while the displacement was measured from DIC results at a location defined hereafter. Theoretically the energy dissipation could be calculated with:

$$
\frac{d W}{d N}=\int F d u
$$

in which $F$ represents the total force on the specimen and $u$ represents the displacement values in the direction parallel to the load direction in the far field where the displacement distribution is nearly uniform.

However, it is not possible to measure the dissipated energy with the displacement directly from the far field away from the crack area. For the reason that the loaddisplacement curves for loading and unloading during one fatigue cycle could be approximately coincide, such that the hysteresis loop could not be observed. This is because the amount of plastic dissipation in one cycle is very small if it is compared with the total strain energy change in single loading or unloading. Moreover, the plastic dissipation only has strong effects on a small local area around crack tip, but in the far field the effects could be negligible. Therefore, in this study the displacement measurements were made around crack tip area.

Some "virtual extensometers" were used, which could be applied in the DIC post-processing software, in the post-processing procedure to obtain local displacement values. The "virtual extensometers" were located at crack tip area, perpendicular to the crack plane and with a length of $1 \mathrm{~mm}$. Meanwhile, the central points of the "virtual extensometers" should approximately coincide with the crack tip point. The displacement values used in the load-displacement hystereses are defined as the elongation of the "virtual extensometers" at the given locations mentioned above on the specimens, and as shown in Fig. 3. 


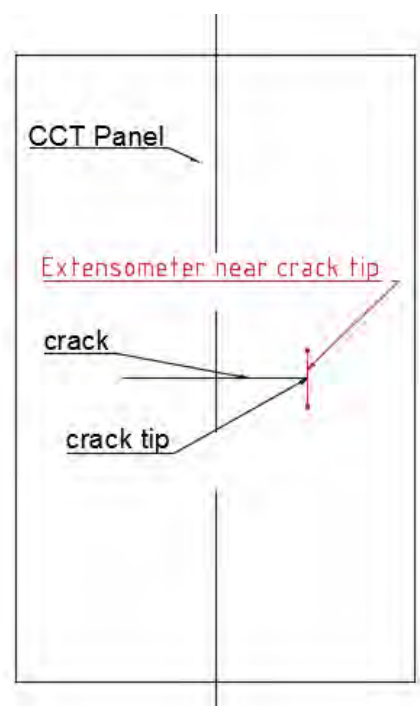

Fig 3. The extensometer at crack tip area to obtain the displacement values in hysteresis loops for measuring the dissipated energy

However, the energy dissipation measured with the displacement values at crack tip area is not the total energy dissipation during fatigue crack growth. In this case, a correction factor $\alpha$ is induced to obtain the total energy dissipation value. The correction factor $\alpha$ is defined as the ratio of energy dissipation calculated with far field displacement, which represents the total energy dissipation during one fatigue cycle, to the energy dissipation calculated from the displacement measurement by extensometers at crack tip area. The value of the correction factor $\alpha$ changes with different crack lengths and various load cases. The value of the correction factor $\alpha$ could be determined by either analytical method or numerical method. In this paper, the value of $\alpha$ was obtained with finite element method. It is assumed that the value of $\alpha$ in the fatigue experiment is approximately equal to the value of $\alpha$ obtained in the corresponding finite element model.

The finite element analysis (FEA) is performed with the commercial finite element software ABAQUS. In this paper, the $2 \mathrm{D}$ plane stress model is chosen to simulate the fatigue experiments. The FEA mesh of this model is shown in Fig. 4.

The FEA model is chosen to be $1 / 4$ model because the symmetricity of Mode I CCT specimen. Then the symmetric boundary conditions are applied on the symmetric edges, except for the crack surface which is considered as a free surface. In order to take the crack closure effect into account, a discrete rigid line is positioned at the position of the crack surface, and the vertical hard contact and tangential frictionless contact properties are chosen to simulate the possible contact of the crack surface caused by crack closure, following the same procedures in [9]. The material properties are simulated with the nonlinear kinematic hardening model in ABAQUS, using the full range stress-strain relation for 2024-T3 obtained from [18]. In this paper the simulation method follows the similar way as the simulation work presented in [1-6,12]. In the FEA model, a stationary crack with no crack propagation is used to model the fatigue crack because the crack growth in one fatigue cycle is very small, and its influence is quite limited and could be neglected. Two fatigue cycles are simulated. The first cycle is used for generating an initial residual plastic field as the plastic energy dissipation becomes stable afterwards. The second cycle is used to obtain both the value of plastic dissipation and the value of the correction factor $\alpha$. Then the value of $\alpha$ in various load cases was used as a correction factor to obtain the total energy dissipation value during the fatigue experiment.

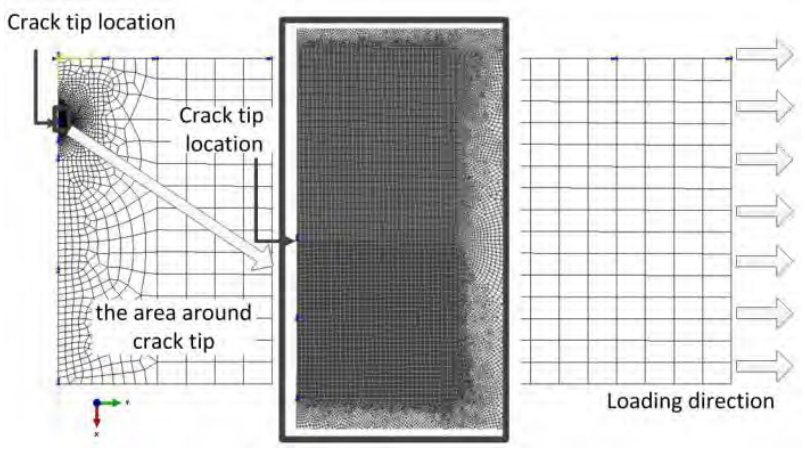

Fig. 4. The FEA model of simulation

As is shown in Fig. 4, a very fine mesh was applied around the crack tip area where the plastic deformation during one cycle is significant. The element size of the crack tip area is around one-tenth of the reverse plastic zone size during the simulated fatigue cycles. And the size of the fine mesh part should be large enough to make sure that all the elements in the plastic zone fall within the fine mesh part. In the field which is far away from the crack tip, a coarser mesh is used to reduce calculation time. The element type used are 2D fournodes bilinear quadrilateral elements with reduced integration. The simulation results of the shapes of the forward plastic zone and the reverse plastic zone in the first fatigue cycle of 2024-T3 specimen at $R=0.05$ and $\Delta K=15 \mathrm{MPa} \sqrt{\mathrm{m}}$ are shown in the Fig. 5 .

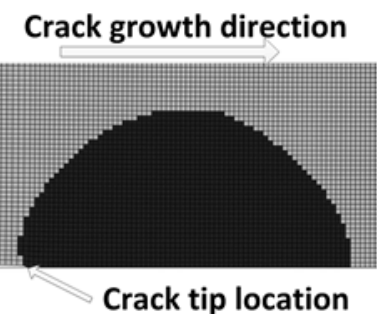

(a)

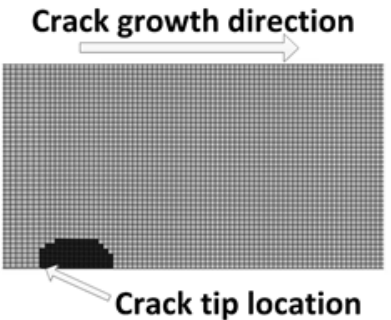

(b)
Fig 5. The 2024-T3 specimen at $R=0.05$. FEA results of (a) the shape of the forward plastic zone at the $1^{\text {st }}$ cycle (b) the shape of the reverse plastic zone at the $1^{\text {st }}$ cycle.

\section{EXPERIMENTAL RESULTS AND DISCUSSION}

\subsection{Experimental results}

In this part, the raw experimental results are presented.

The fatigue tests were done with an initial crack length $2 a \approx 16 \mathrm{~mm}$. The fatigue crack length $a$ at different 
number of cycles with different stress ratios is shown in Fig. 6.

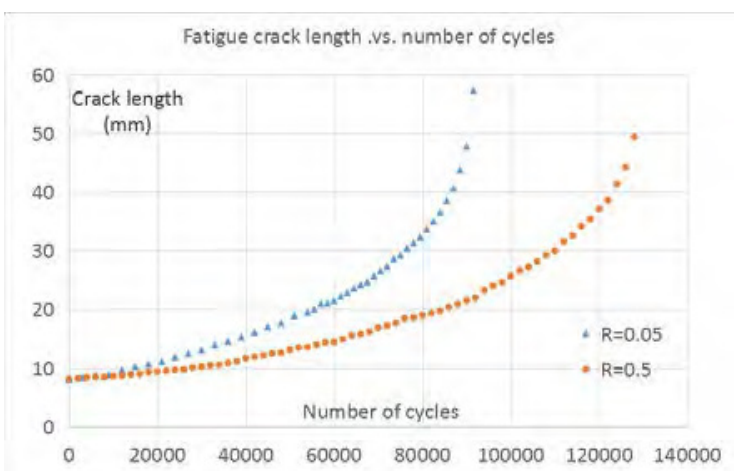

Fig. 6. The crack length versus number of cycles in different stress ratios

The energy dissipation per cycle $d W / d N$ was obtained for about every $1 / 10$ of $N_{f}$, where $N_{f}$ is defined as the total fatigue life of the specimen. The trend is shown in Fig. 7.

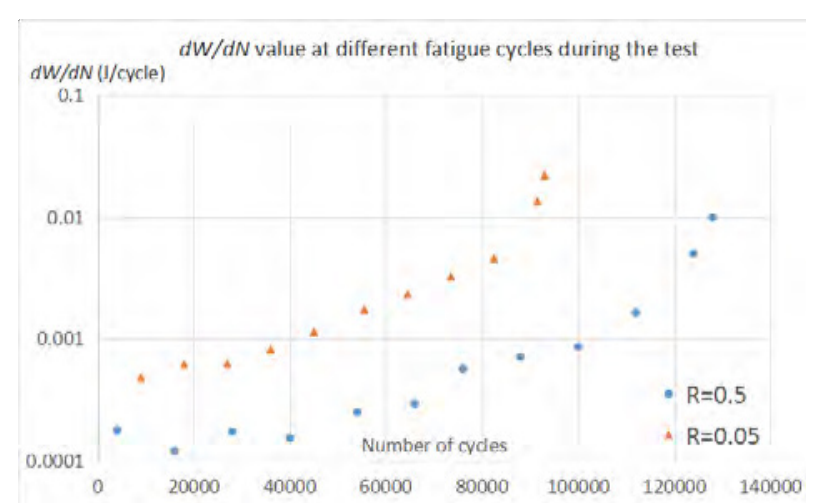

Fig. 7. $d W / d N$ versus number of cycles in different stress ratios

\subsection{Discussion based on experimental results}

\subsection{1 da/dN versus $\Delta K$ and $d W / d N$ versus $\Delta K$}

The fatigue crack growth rate are shown against $\Delta K$ in Fig. 8. This figure shows that the $d a / d N$ data could approximately fit the Paris relation, although there is some error. The Paris relations for the curves are

for $\mathrm{R}=0.05$ :

$$
\frac{d a}{d N}=1.6499 \times 10^{-7}(\Delta K)^{2.70844}
$$

for $\mathrm{R}=0.5$ :

$$
\frac{d a}{d N}=1.9704 \times 10^{-7}(\Delta K)^{2.90346}
$$

The energy dissipation per cycle for both $\mathrm{R}=0.5$ and $\mathrm{R}=0.05$ is plotted against $\Delta K$ in Fig. 9 .

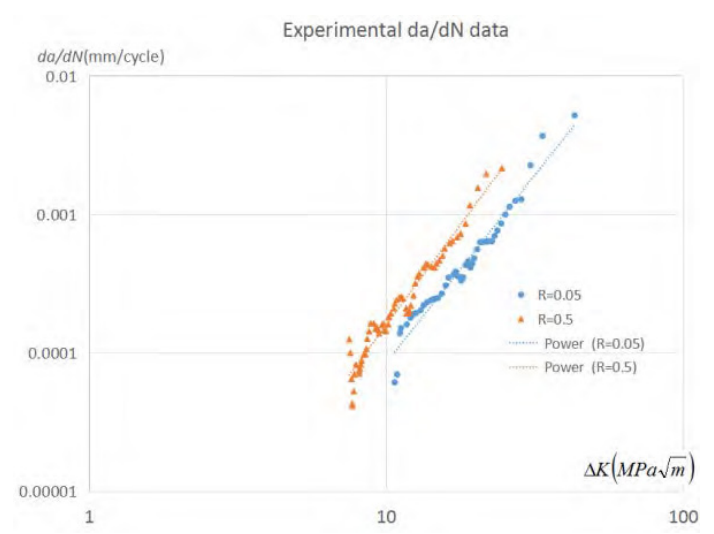

Fig. 8. $d a / d N$ versus $\Delta K$ for 2024-T3

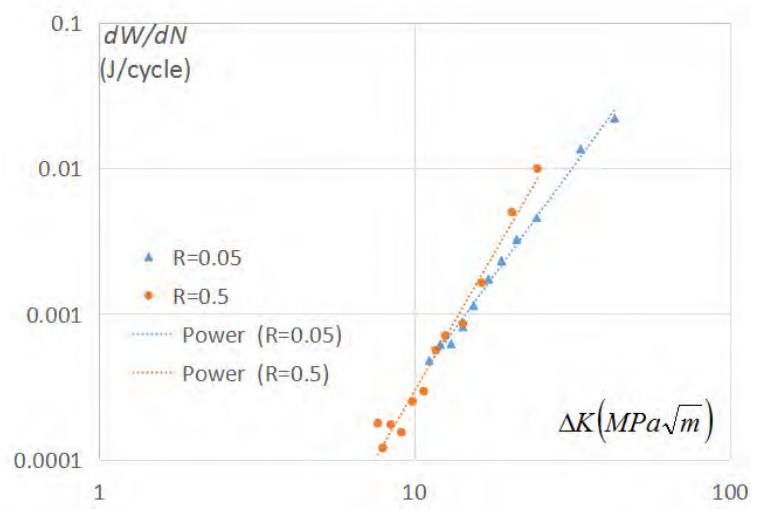

Fig. 9. The experimental energy dissipation per cycle $d W / d N$ versus $\Delta K$

From Fig. 9, it could been observed that the $d W / d N$ versus $\Delta K$ curves of both $\mathrm{R}=0.5$ and $\mathrm{R}=0.05$ seems to fall into the same scatter band, which agrees with [12]. The power law fit for the data in Fig. 9 are for $\mathrm{R}=0.05$ :

$$
\frac{d W}{d N}=3.48547 \times 10^{-7}(\Delta K)^{2.97284}
$$

for $\mathrm{R}=0.5$ :

$$
\frac{d W}{d N}=5.30572 \times 10^{-8}(\Delta K)^{3.74486}
$$

\subsection{2 dW/da during fatigue crack propagation}

From Eq.(1) it is known that the value of the $d W / d a$ is equal to $d W / d N$ divided by $d a / d N$. So in Fig. 10, the energy dissipation per cycle $d W / d N$ versus fatigue crack growth rate $d a / d N$ is plotted. For nearly constant values of $d W / d a$, the curves in Fig. 10 should be nearly straight lines. Thus the curves could be fitted to a power law whose exponent is close to one. The resulting equations after curve fitting in Fig. 10 are

for $\mathrm{R}=0.05$ :

$$
\frac{d a}{d N}=0.1563\left(\frac{d W}{d N}\right)^{0.9277}
$$

for $\mathrm{R}=0.5$ :

$$
\frac{d a}{d N}=0.0773\left(\frac{d W}{d N}\right)^{0.7573}
$$




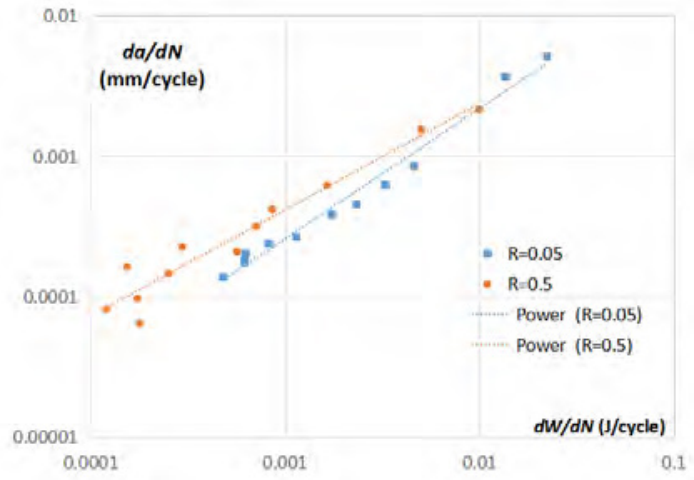

Fig. 10. $d a / d N$ versus $d W / d N$

However, from Eq.(7) and Eq.(8) it could be concluded that both curves in Fig. 10 are not linear, which means the $d W / d a$ does not remain constant for the tested stress ratios. Moreover, the curve of $\mathrm{R}=0.5$ is clearly nonlinear, while the curve of $\mathrm{R}=0.05$ is much closer to a linear trend. Therefore, the trend of how $d W / d a$ changes differs for different stress ratios. These results answer the first research question raised in the introduction: $d W / d a$ is not a material property that is approximately constant.

Then the second research question needs to be answered, because it is important for answering whether $d W / d N$ is an effective similitude parameter to evaluate the fatigue crack growth regardless of the influence of stress ratio.

In order to answer the second question raised in the introduction, the $d W / d N$ versus $d a / d N$ data in Fig. 10 for $\mathrm{R}=0.05$ and $\mathrm{R}=0.5$ is compared. It could be observed that for the same $d a / d N$ value, the $d W / d N$ for $\mathrm{R}=0.05$ is larger than $d W / d N$ for $\mathrm{R}=0.5$. Therefore, the $d W / d a$ value of $\mathrm{R}=0.05$ is larger than the value of $\mathrm{R}=0.5$. This is the answer to the second research question: the same value of $d W / d N$ does not mean the same $d a / d N$.

The same result could also be obtained from both Fig. 8 and Fig. 9. For the same $\Delta K$ value, the $d W / d N$ values for both stress ratios seems to be nearly the same, while the fatigue crack growth rate of $\mathrm{R}=0.5$ is much larger than the fatigue crack growth rate of $\mathrm{R}=0.05$. So the $d W / d a$ value of $\mathrm{R}=0.5$ should be smaller. Meanwhile from Fig. 9, it is possible to conclude that the $d W / d N$ could be considered as an equivalent similitude to $\Delta K$, for the $d W / d N$ stays nearly the same at the same $\Delta K$ for both stress ratios. Obviously, the amount of two tests is too small to derive firm conclusions. Hence, more test data at more stress ratios is required to confirm this hypothesis.

\subsubsection{The energy balance equation for the fatigue crack growth phenomenon}

From the discussion above, it could be concluded that the plastic energy dissipation per unit of fatigue crack growth $d W / d a$ is not an effective parameter to evaluate the fatigue crack growth rate, because the same $d W / d N$ could lead to different $d a / d N$ values. However, is there any reason to explain this conclusion?
Here the phenomena of crack propagation is considered at a more fundamental level from a physical point of view. The analysis here is started from the example of the static crack propagation. Irwin and Orowan proposed that crack resistance is equal to the sum of the surface energy and the plastic strain work accompanying crack extension [19].

In a similar way, then the static crack propagation could be thought of as energy conservation. The energy balance equation of the crack propagation will then be:

$$
\dot{W}=\dot{U}_{a}+\dot{U}_{p l}+\dot{U}_{e}
$$

The physical meaning of the equation in differential form is that: the external work done to the solid $\dot{W}$ is equal to the sum of the energy cost by the formation of the new crack surface $\dot{U}_{a}$, the plastic energy dissipation $\dot{U}_{p l}$, and change of the elastic strain energy of the solid $\dot{U}_{e}$. That also agrees with the opinion of Xiao [20]: the surface-forming energy release is equal to the power of the external force minus the rate of the stored elastic strain energy and the rate of dissipation by plastic deformation. The three variables in the right side of the equation are coupled. But the crack propagation is only directly related to $\dot{U}_{a}$, although the other two variables would change as $\dot{U}_{a}$ changes, because $\dot{U}_{p l}$ and $\dot{U}_{e}$ are only the consequences rather than the reasons or driving force of the crack propagation. For metals, the value of the $\dot{U}_{p l}$ is significantly larger than $\dot{U}_{a}$ and $\dot{U}_{e}$.

Then the Eq.(9) is extended to the fatigue crack growth situation. When the equation is integrated over one fatigue cycle, it becomes:

$$
\frac{d W}{d N}=\frac{d U_{a}}{d N}+\frac{d U_{p l}}{d N}+\frac{d U_{e}}{d N}
$$

The physical explanation of the equation above is that the external work done (mainly by fatigue loading here) during one cycle $d W / d N$ is equal to the sum of the energy dissipation for the fatigue crack propagation $d U_{a} / d N$, the plastic dissipation during one cycle $d U_{p l} / d N$, and the change of the elastic strain energy after one cycle $d U_{e} / d N$. In this concept, the external work represents a driving force for fatigue crack growth, while the energy storage and dissipation over the created crack surface represents effectively the resistance against crack propagation. Both the driving force and resistance depend on the applied fatigue load cycle, the geometry of the problem, crack length and the material properties.

Similar to the static situation, the three variables on the right hand side of the equation are coupled. The fatigue crack propagation is only directly related to $d U_{a} / d N$, while $d U_{e} / d N$ and $d U_{p l} / d N$ are only the consequences of the fatigue crack propagation. The value of the $d U_{p l} / d N$ is much larger than $d U_{a} / d N$ and $d U_{e} / d N$ for metallic materials. Therefore, in general it holds approximately that:

$$
\frac{d W}{d N} \approx \frac{d U_{p l}}{d N}
$$

The term $d U_{a} / d N$ is directly linked to the fatigue crack growth, which is related to the fatigue crack growth rate and fatigue crack surface characteristics. The fatigue crack surface characteristics are influenced by the mechanics of fatigue crack propagation and could be 
represented by the roughness of the crack surface. The term $d U_{p l} / d N$ should be established based on the entire load cycle, including the forward plastic zone development during loading and the formation of the reverse plastic zone during unloading.

Now the answer to the question of the varying value of $d W / d a$ among different load cases is clear: the correct similitude to describe the fatigue crack propagation is $d U_{a} / d N$ rather than $d U_{p l} / d N$, and the plastic energy dissipation is only the consequence accompanying the fatigue crack growth. The change of the ratio of $d U_{a} / d N$ to $d U_{p l} / d N$ leads to the variance of $d W / d a$.

However, the quantity of the $d U_{a} / d N$ value is too small to be measured with fatigue experiments. And it is also impractical to obtain the $d U_{a} / d N$ value for its extremely small value compared with $d U_{p l} / d N$. So for further research and engineering practices, the value of $d U_{p l} / d N$ seems to be the only choice if there are no extraordinary methods to quantify $d U_{a} / d N$ for real tests. But extra care has to be taken since $d U_{p l} / d N$ is not directly related to $d a / d N$, and that $d W / d a$ cannot be considered as constant or as a material property.

\subsubsection{Limitations of the work in this paper}

First, there are some shortcomings with the experimental study in this paper. Only 2 stress ratios were tested and only a few $d W / d N$ data points were obtained for each stress ratio. While this is enough for the first step of the study to observe a trend, it is not sufficient to come to firm conclusions. Therefore more stress ratios will be tested and more data points will be obtained in future work. Besides, the $d W / d N$ is obtained by a combination of experimental measurements and FEA calculation, such that errors in numerical simulation might affect the final results.

Second, both the cases in fatigue experiments and FEA simulations are assumed to be pure Mode I. However, in reality it is not strictly true, because the fatigue crack direction is not strictly transverse to the load direction and crack surfaces are not smooth and plain. Therefore, the crack is in mixed Mode I-II-III rather than pure Mode I, which would lead to more energy dissipation than currently considered. Meanwhile, the actual crack surface area is much larger than assumed by taking the of crack length $a$ multiplied by thickness $t$ because of roughness of the crack surface. Hence, that will result in larger $d U_{a} / d N$ and $d U_{p l} / d N$ values than assumed in both experimental and numerical cases. For the experimental study, the energy dissipation measured is not the theoretical energy dissipation of pure Mode I, and in the FEA modelling those effects cannot be simulated and would result in some error in the simulation.

Moreover, the stress and strain distributions are not uniform through the thickness direction of the specimen, therefore the energy dissipation properties are not the same for the through-the-thickness direction. On the mid-plane of the specimen, it is more like plane strain, but on the surface of the specimen, it is more like plane stress. Nevertheless, in this paper for both experimental and numerical study, it is assumed to be nearly uniform through thickness direction for the thickness is relatively small compared with other geometry parameters: the thickness effect is ignored. In fatigue experiments, the displacement measured on the surface is chosen to represent the whole specimen, and in FEA only the 2D model is applied.

Finally, there must be some error in the FEA modelling. Besides neglecting the rough crack surface, curved crack path and the differences through thickness direction mentioned above, the FEA model still brings in some errors. For one thing, in the simulations, a stationary crack is used, so it fails to illustrate the influence of residual plastic wake behind crack tip and the effect of crack propagation on the energy dissipation. For another, the material model used and the material data from [18] could be a little different from the material properties of the specimens used, based on material production variability.

Therefore, there is still a lot of work to be done in the future. More experimental data at various stress ratios should be gathered, and more care could be taken on the differences between assumption and reality. Meanwhile, a more detailed numerical study which is closer to reality is also needed in the future work.

\section{CONCLUSION}

In this paper, an experimental study on 2024-T3 fatigue crack growth test is carried out. The plastic energy dissipation at two different stress ratios is measured. The experiment shows that plastic dissipation per unit of fatigue crack growth $(d W / d a)$ does not stay constant for different load cases. Therefore the value of $d W / d a$ cannot be regarded as a material property, and cannot be used directly to predict $d a / d N$.

The reason is that the $d U_{p l} / d N$ is not an effective similitude parameter. It is the crack surface forming energy that is directly linked to fatigue crack growth, which is also the proper similitude parameter rather than plastic dissipation. The plastic dissipation is only the consequence which accompanies the fatigue crack growth. However, the value of $d U_{a} / d N$ is too small to be measured accurately in experiments. Then, the plastic dissipation seems to be the only choice whose value could be obtained in reality. But it should be aware that plastic dissipation does not directly link to fatigue crack growth and some extra efforts are needed to bridge this gap.

There are still a lot of limitations in this paper, which result from the differences between assumption and reality. So some future work is still needed to overcome the shortcoming of the current work.

Finally, if we consider the contradiction between the work of [1-6,15] and [12-13], this paper is on the side of [12-13] stating that $d W / d a$ is variable. Considering that in the work done by Karlsson et al. [7-11] and Zheng[14] the definitions of $d W / d N$ are slightly different, further numerical details need be given in order to make a comparison between their work and the work in this paper. 


\section{References}

1. J.S. Daily, N. W. Klingbeil, Int J Fatigue, 32 (2010) 1710-1723.

2. C.M. Baudendistel, N.W. Klingbeil, Int J Fatigue, 51 (2013) 96-104.

3. J.S. Daily, N.W. Klingbeil, Int J Fatigue, 28 (2006) 1725-1738.

4. J.S. Daily, N.W. Klingbeil, Int J Fatigue, 26 (2004) 727-738.

5. N.W. Klingbeil, Int J Fatigue, 25 (2003) 117-128.

6. N. Klingbeil1, J. Daily, C. Baudendistel. Key Eng Mater, 378-379 (2008) 385-404.

7. D. Cojocaru, A.M. Karlsson, ADV ENG SOFTW, 39 (2008) 995-1009.

8. D. Cojocaru, A.M. Karlsson, Int J Fatigue, 31 (2009) 1154-1162.

9. P.G. Nittur, A.M. Karlsson, L.A. Carlsson, Eng Fract Mech, 124-125 (2014) 155-166.

10. P.G. Nittur, A.M. Karlsson, L.A. Carlsson, Int J Fatigue, 54 (2013) 47-55.

11. G. Ding, A.M. Karlsson, M.H. Santare, Int J Fatigue, 94 (2017) 89-96.

12. N. Ranganathan, F. Chalon, S. Meo, Int J Fatigue, 30 (2008) 1921-1929.

13. M. Mazari, B. Bouchouicha, M. Zemri, M. Benguediab, N. Ranganathan, Comput Mater Sci, 41 (2008) 344-349.

14. X. Zheng, H. Cui, X. Su, C.C. Engler-Pinto Jr., W. Wen, Eng Fract Mech, 114 (2013) 151-165.

15. K.V. Smith, Eng Fract Mech, 78 (2011) 3183-3195.

16. M. Besel, E. Breitbarth, Int J Fatigue, 93 (2016) $92-$ 108.

17. E. Breitbarth, M. Besel, Int J Fatigue, 100 (2017) 263-273.

18. Military Handbook - MIL-HDBK-5H: Metallic Materials and Elements for Aerospace Vehicle Structures (Knovel Interactive Edition), U.S. Department of Defense.

19. M. Janssen, J. Zuidema, R. Wanhill, Fracture Mechanics, Second Edition: Fundamentals and Applications, vol. $2^{\text {nd }}$ ed, CRC Press, 2004, Page 96.

20. S. Xiao, H. Wang, B. Liu, K. Hwang, J Mech Phys Solids, 84 (2015) 336-357. 